\title{
FLUXO SUBSUPERFICIAL INTERCEPTADO POR ESTRADA RURAL: CARACTERÍSTICAS E DISTRIBUIÇÃO NA PAISAGEM
}

\author{
SUBSURFACE FLOW INTERCEPTED BY RURAL ROADS: \\ CHARACTERISTICS AND DISTRIBUTION ON THE LANDSCAPE
}

\author{
FLUJO SUBSUPERFICIAL INTERCEPTADO POR CARRETERA \\ RURAL: CARACTERÍSTICAS Y DISTRIBUCIÓN EN EL PAISAJE
}

Márcia Cristina da Cunha - Universidade Estadual do Centro Oeste - Guarapuava - Paraná - Brasil marcia1cunha@yahoo.com.br

\author{
Edivaldo Lopes Thomaz - Universidade Estadual do Centro Oeste - Guarapuava - Paraná - Brasil \\ edilopes.thomaz@gmail.com
}

\section{Resumo}

Embora as estradas rurais permitam o desenvolvimento das comunidades rurais, elas alteram a resposta hidrológica natural da bacia hidrográfica. Esse estudo foi realizado na bacia do Rio Guabiroba, GuarapuavaPR, com o objetivo de identificar e caracterizar os tipos de interceptação de fluxo subsuperficial causados por cortes de estradas rurais associados à sua distribuição espacial ao longo da bacia hidrográfica. Para tanto, como metodologia utilizada foram realizados levantamentos de campo com observação direta para determinar as áreas de interceptação do fluxo em subsuperfície e sua distribuição na bacia do Rio Guabiroba. Três tipos principais foram identificados e denominados como: fluxo de retorno, fluxo pipe e fluxo insaturado. 0 fluxo de retorno é frequentemente distribuído próximo aos rios e no fundo de vale, o fluxo pipe está presente principalmente em média vertente, e o fluxo insaturado ocorre mais frequentemente em encostas superiores, expelido nos taludes mais profundos. Conclui-se, portanto, que os tipos de interceptação subsuperficial e a distribuição espacial desses podem alterar a dinâmica hidrológica natural da bacia, provocando um tempo de resposta menor que por meio da infiltração e deslocamento no solo.

Palavras-chave: Bacia hidrográfica rural, resposta hidrológica, conectividade, área saturada.

\begin{abstract}
Although rural roads enable the development of rural communities, they change the natural hydrological response of the catchment. This study was carried out in the Guabiroba River Basin, Guarapuava-PR (Brazil), with the objective of identifying and characterizing the types of subsurface flow interception caused by rural road cuts associated with their spatial distribution along the hydrographic basin. For this, as methodology used, we conducted a field survey with direct observation to determine the areas of interception of subsurface flow and their distribution in the catchment Guabiroba River basin. Three major types were identified: return flow, pipe flow, and saturated troughflow. Return flow is often distributed in rivers and terraces, pipe flow is present mainly in midslopes, and saturated troughflow occurs most often in upper slopes, expelled into the deepest embankments. It is concluded, therefore, that the types of subsurface interception and their spatial distribution can alter the natural hydrological dynamics of the basin, provoking a shorter response time than through infiltration and displacement in the soil.
\end{abstract}

Keywords: Rural catchment, hydrologic response, connectivity, saturated area. 


\section{Resumen}

Pese a que las carreteras rurales permiten el desarrollo de las comunidades rurales, cambian la respuesta hidrológica natural de la cuenca. Este estudio fue realizado en la cuenca del Río Guabiroba, Guarapuava-PR, con el objetivo de identificar y caracterizar los tipos de interceptación de flujo subsuperficial causados por cortes de carreteras rurales asociadas a su distribución espacial a lo largo de la cuenca hidrográfica del Río Guabiroba. Para ello, como metodología utilizada hemos desarrollado un estudio de campo con observación directa para determinar las zonas de captura del flujo en el subsuelo y su distribución en la cuenca. Se identificaron tres tipos principales: flujo de retorno que, es frecuentemente distribuido próximo los ríos e al fondo del valle, el flujo pipe, que está presente principalmente en la media vertiente, y el flujo no saturado, que se produce más a menudo en laderas superiores, segregado en inclinaciones más profundas. Se concluye, por lo tanto, que los tipos de interceptación subsuperficial y la distribución espacial de éstos, pueden alterar la dinámica hidrológica natural de la cuenca, provocando un tiempo de respuesta menor que por medio de la infiltración y desplazamiento en el suelo.

Palabras clave: Cuenca rural, la respuesta hidrológica, conectividad, área saturada.

Introdução

As estradas rurais são fundamentais para o desenvolvimento local em áreas rurais (Faiz et al., 2012), pois garantem a melhoria da qualidade de vida da população e, muitas vezes, essa é a única forma de acesso que a população tem aos serviços básicos disponibilizados em área urbanas, como saúde, educação, lazer, trabalho e outros (Bryceson; Bradbury; Bradbury, 2008; Faiz et al., 2012). Além disso, muitas vezes, a rede viária é construída em diferentes formas do relevo, tais como áreas montanhosas, encostas, terraço fluvial e próximo ao sistema de drenagem. A distribuição dessas vias em diferentes setores da vertente e sua tendência em cruzar a rede de drenagem afetam o movimento da água e sedimento na bacia (Luce; Wemple, 2001; Chappell, 2010).

Assim, as estradas têm efeitos positivos e negativos. De um lado, são importantes para o desenvolvimento socioeconômico, especialmente nos países em desenvolvimento (Faiz et al., 2012; Sidle; Ziegler, 2012). Por outro lado, podem causar vários impactos sobre o ecossistema, tais como mudanças nos processos hidrogeomorfológicos, escoamento superficial, interceptação do fluxo subsuperficial, produção e transferência de sedimentos em sistemas aquáticos (Luce, 2002; Baartman et al., 2013; Thomaz; Vestena; Ramos-Scharrón, 2014).

Recentes tentativas têm sido feitas para tornar as estradas mais harmoniosas com o meio ambiente e, ao mesmo tempo, a integração dos princípios estéticos para torná-las mais atraentes para os usuários (Bryceson; Bradbury; Bradbury, 2008; Cheng et al. 2015). Apesar disso, por um longo período, as estradas rurais foram reconhecidas como uma 
importante fonte de escoamento superficial e produção de sedimentos e desempenhavam também um papel fundamental conexão entre vertente e rio (Jones, 1981; Sidle; Ziegler, 2012).

O comportamento hidrológico de estradas é a chave para compreensão dos seus efeitos na dinâmica de bacias hidrográficas. Embora as estradas apresentem problemas muito específicos no campo da hidrogeomorfologia de vertente, elas também representam uma grande oportunidade para o avanço da ciência, enquanto solução de importantes problemas ambientais, auxiliando no entendimento dos processos em bacias hidrográficas, que vão desde escala local até escala global (Bryan; Jones, 1997; Wemple; Swanson; Jones, 2001; Cunha; Thomaz, 2015).

Em bacias hidrográficas, as estradas rurais são foco de estudos hidrogeomorfológicos, baseados no escoamento superficial, produção de sedimentos transferência deste para o rio (Luce, 2002; Thomaz; RamosScharrón, 2015). No entanto, uma abordagem para a identificação e caracterização dos tipos de fluxo subsuperficial interceptados por cortes de estradas rurais e sua distribuição espacial ao longo da bacia hidrográfica é escassa (Bryan; Jones, 1997; Luce, 2002).

A identificação e caracterização dos tipos de interceptação de fluxo subsuperficial são importantes para a compreensão de seus mecanismos ativos, particularmente nas áreas em que as estradas são abandonadas ou com baixa frequência de uso e manutenção (Sidle; Ziegler, 2012). Além disso, as pressões antropogênicas e a organização da paisagem são fundamentais para a pesquisa em ciências geográficas. Portanto, o objetivo deste estudo foi identificar e caracterizar os tipos de interceptação de fluxo subsuperficial, causados por cortes estradas rurais, associados à sua distribuição espacial ao longo da bacia hidrográfica.

Material e métodos

\section{Área de estudo}

Este estudo foi realizado na bacia do Rio Guabiroba, em Guarapuava, Estado do Paraná, Brasil (Figura 1). A bacia possui uma área de $24 \mathrm{~km} 2$ (2,401 ha), a hierarquia fluvial é de 4a ordem em escala de 1:10.000. A bacia é composta somente de estradas rurais, sem pavimentação, com $130 \mathrm{~km}$ de vias. A litologia predominante da área de estudo é basalto da Formação São 
Bento (ATLAS geológico do estado do Paraná, 2001). Quatro tipos de solo estão presentes na cobertura superficial: Latossolo, Cambissolo, Neossolo, Gleissolo (IUSS Working Group WRB, 2006). Além disso, ocorre também em algumas unidades o afloramento de rochas na vertente.

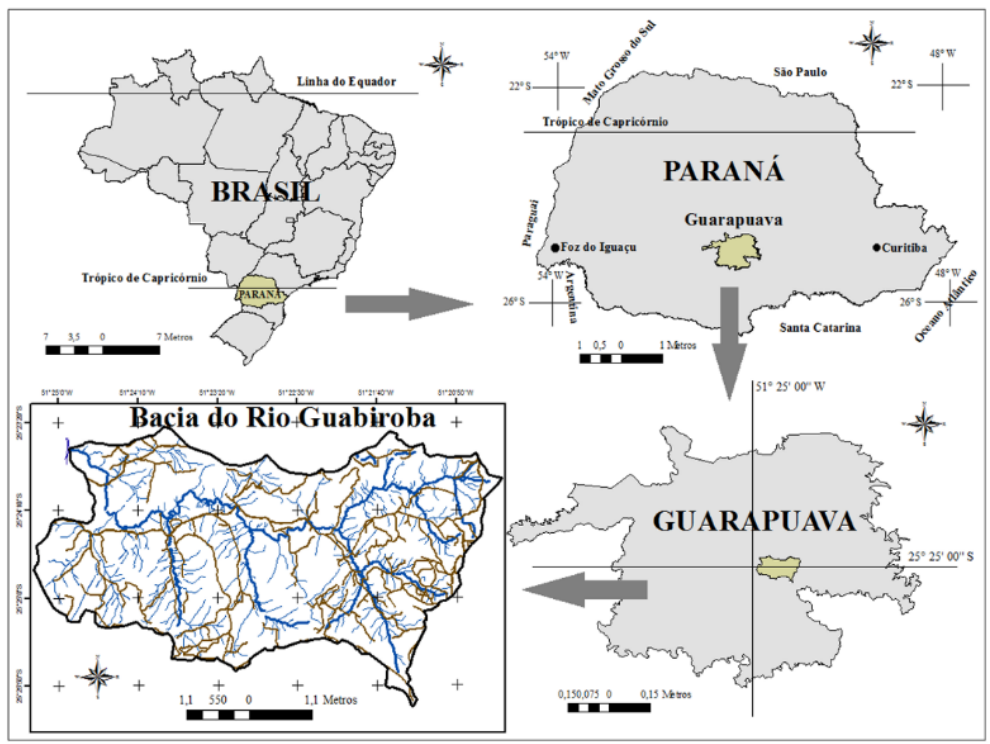

Figura 1 - Localização da área de estudo em relação ao município de Guarapuava Fonte: Elaborado pelos autores (2017).

Aproximadamente $75 \%$ da área de estudo têm a inclinação entre $12 \%$ e mais de $30 \%$. Köppen (1948) classificou o clima na área de estudo como mesotérmico subtropical úmido (Cfa). A temperatura média anual é de $17-18^{\circ} \mathrm{C}$. A precipitação é de $1.800-2.000 \mathrm{~mm}$ e a evapotranspiração anual é de 900-1.000 mm (Caviglione et al., 2000). O uso do solo não é diversificado, consistindo principalmente de floresta secundária (53,9\%), pastagem $(19,9 \%)$ e reflorestamento $(11,3 \%)$.

Espacialização e caracterização de tipos de interceptação de fluxo subsuperficial

No estudo, optou-se por um esquema teórico denominado empíricocartográfico-monitoramento (Figura 2). 


\section{Revisão Bibliográfica:}

Leituras e observações sobre espacialização e caracterização de fluxo em subsuperfície: bacia de drenagem, vertente, canal fluvial, estradas rurais.

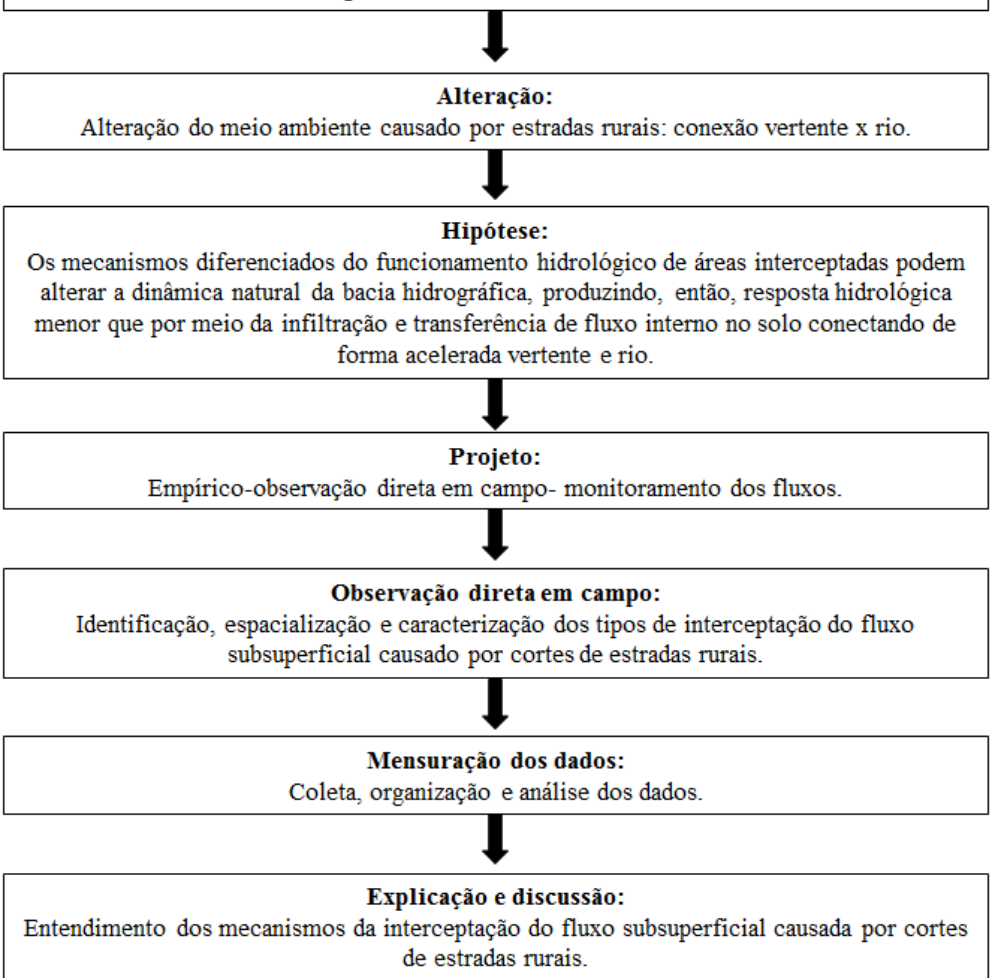

Figura 2 - Esquema teórico empírico-cartográfico e de monitoramento

Fonte: Organizado pelos autores (2017).

Para a espacialização e caracterização dos tipos de interceptação do fluxo subsuperficial causados por cortes de estradas rurais, em um primeiro momento, foi realizada a identificação preliminar das áreas de interceptação em subsuperfície. Percorreu-se ao todo $130 \mathrm{~km}$ de estradas rurais na área de estudo (total viário). O objetivo foi observar e identificar inicialmente locais potenciais para interceptação de fluxo subsuperficial por cortes de estradas rurais, por exemplo, locais com umidade e afloramento de água no talude e na estrada, presença de vegetação como samambaias ou juncos (plantas que crescem em lugares úmidos), dutos 
em atividade nos períodos de chuva e áreas próximas ao fundo de vale (Figura 3). As observações ocorreram logo após os eventos pluviométricos (em média de 10 a 20 min depois da chuva), com o intuito de separar o fluxo superficial do fluxo subsuperficial.
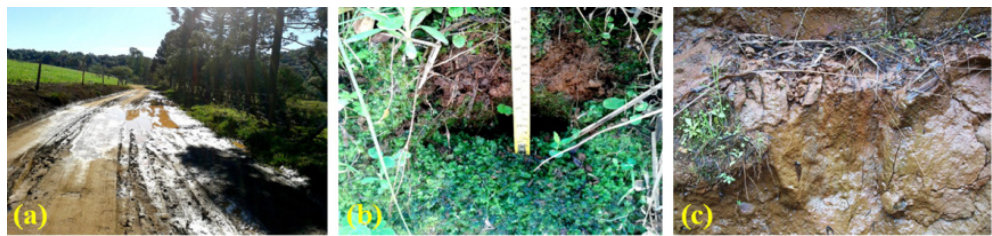

Figura 3 - Identificação das áreas potenciais para interceptação do fluxo subsuperficial por estradas rurais. Em (a) área saturada, em (b) pipe em atividade, em (c) água exfiltrada no talude da estrada

Fonte: Organizado pelos autores (2017).

Na sequência, foram especializadas as áreas de interceptação do fluxo em subsuperfície. Do total de estradas percorridas, foram registrados 20 locais de interceptação do fluxo subsuperficial causados por cortes das estradas rurais. Foi contatado que as áreas potenciais de interceptação do fluxo subsuperficial estavam distribuídas em diferentes setores da vertente, sendo os mais comuns em três seções, fundo de vale, média e alta vertente. Nesta fase, foram observados também a frequência dos tipos de interceptação do fluxo subsuperficial.

Verifica-se também, as características de cada tipo de interceptação do fluxo com as áreas onde eles foram localizados: localização na vertente (baixa, média ou alta vertente), forma do terreno (côncavo, convexo ou retilíneo), posição na vertente (relevo plano, ondulado ou montanhoso).

Para a medição dos locais de intercepção ao longo do sistema viário foi utilizado o Sistema de Posicionamento Global (GPS) e a Base Digital Cartográfica em uma escala de 1:30.000, disponibilizada pela Prefeitura Municipal de Guarapuava. O mapeamento da paisagem com a distribuição espacial das áreas de interceptação do fluxo subsuperficial foi realizado com um Sistema de Informações Geográficas (SIGs), Quantum GIS versão 2.18.5.

Jairosi (2001) informa que, com relação aos dados geoespaciais, a padronização de simbologia (feições) pode trazer resultados como o crescimento mútuo do entendimento do dado geográfico em diversos usuários colaborando com o processo cognitivo. 
Portanto, os diferentes tipos de interceptação foram identificados e caracterizados por nomenclatura específica, como segue: 1- fluxo de retorno, 2- fluxo pipe e 3- fluxo insaturado. Esta designação foi baseada na observação direta com as características que cada fluxo subsuperficial apresentou (Anderson; Burt, 1990; Knighton, 1998). Destaca-se, ainda, que as representações constantes no trabalho estão de acordo com as normas contidas no Manual Técnico T 34-700, o qual foi elaborado pela Diretoria do Serviço Geográfico do Exército Brasileiro (2000).

\section{Resultados}

\section{Espacialização das áreas de interceptação do fluxo subsuperficial}

O total de cada tipo de interceptação e o tempo de permanência de cada fluxo foi diferente em cada setor (Tabela 1). No total de $130 \mathrm{~km}$ estradas percorridas foram identificadas 20 áreas de interceptação do fluxo subsuperficial. Como mencionado nos materiais e métodos, a nomenclatura foi designada como: fluxo de retorno (7 locais identificados), fluxo pipe (5 locais identificados) e fluxo insaturado (8 locais identificados). Essas áreas de intercepção estão distribuídas em diferentes setores topográficos na bacia hidrográfica estudada (Figura 4).

\begin{tabular}{|c|c|c|c|c|c|}
\hline $\begin{array}{c}\text { Tipo de } \\
\text { interceptação }\end{array}$ & $\begin{array}{c}\text { Localização na } \\
\text { vertente }\end{array}$ & $\begin{array}{c}\text { Forma do } \\
\text { terreno } \\
\text { predominante }\end{array}$ & $\begin{array}{c}\text { Posição na } \\
\text { paisagem }\end{array}$ & $\begin{array}{c}\text { Total das } \\
\text { áreas de } \\
\text { interceptação } \\
\text { observadas }\end{array}$ & $\begin{array}{c}\text { Tempo médio } \\
\text { de fluxo } \\
\text { em dias } \\
\text { consecutivos } \\
\text { após a chuva }\end{array}$ \\
\hline $\begin{array}{l}\text { 1- Fluxo de } \\
\text { retorno }\end{array}$ & $\begin{array}{l}\text { Terraço fluvial, ou } \\
\text { próximo a áreas } \\
\text { úmidas. }\end{array}$ & Côncavo & $\begin{array}{c}\text { Relevo Suave } \\
\text { ondulado }\end{array}$ & 7 & 15 \\
\hline 2- Fluxo pipe & $\begin{array}{c}\text { Taludes das } \\
\text { estradas em } \\
\text { média vertente. }\end{array}$ & Retilíneo & $\begin{array}{l}\text { Relevo } \\
\text { Ondulado }\end{array}$ & 5 & 10 \\
\hline $\begin{array}{l}\text { 3- Fluxo } \\
\text { insaturado }\end{array}$ & $\begin{array}{l}\text { Expelido no } \\
\text { talude da estrada } \\
\text { em alta vertente. }\end{array}$ & Convexo & $\begin{array}{c}\text { Relevo } \\
\text { Montanhoso }\end{array}$ & 8 & 8 \\
\hline
\end{tabular}

Tabela 1 - Espacialização das áreas de interceptação do fluxo subsuperficial 


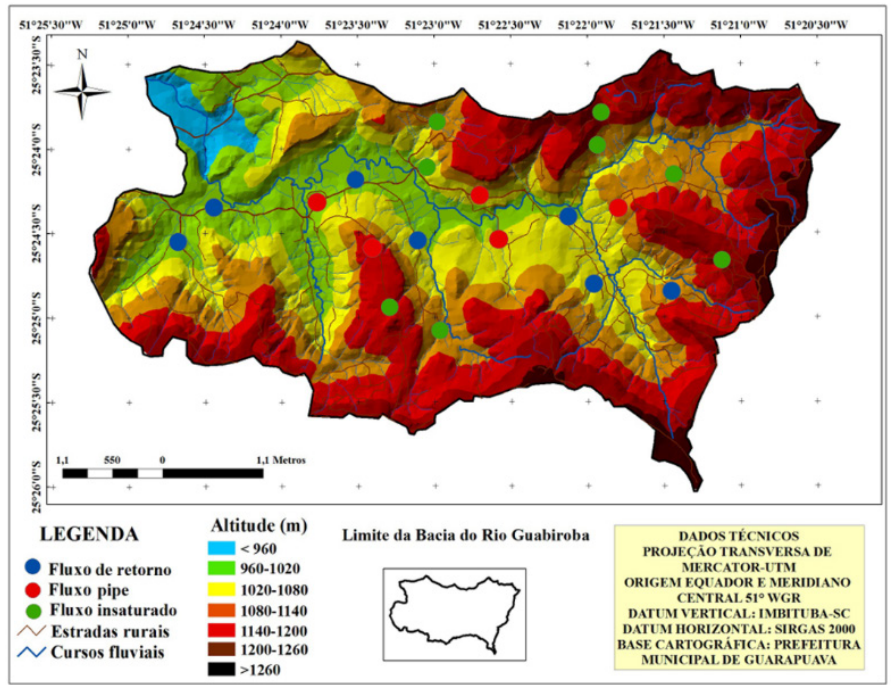

Figura 4 - Espacialização dos tipos de fluxos subsuperficiais que ocorrem em áreas que são interceptadas por cortes de estradas rurais

Fonte: Elaborado pelos autores (2017).

O tempo de permanência do fluxo interceptado refere-se a quantos dias o fluxo permanece em atividade após o cessar da precipitação, ou seja, o tempo de escoamento depois da interceptação causada pela estrada. Foi constatado que esse tempo foi maior no fluxo de retorno, permanecendo em atividade até 15 dias consecutivos após a chuva. Além disso, o tempo de permanência do fluxo diminuiu no fluxo pipe com 10 dias ativos e foi de 8 dias no fluxo insaturado.

O fluxo de retorno prevaleceu no terraço fluvial e próximo a áreas úmidas (fundo de vale) (Figura 5a). Nota-se que esses locais de intercepção são importantes na geração de fluxo de retorno próximo à rede de drenagem. A forma do terreno é convergente-côncavo e foi clara em representar a maneira como os talvegues condicionaram o fluxo subsuperficial em direção às áreas mais baixas desses locais, facilitando o acúmulo e a permanência da água no setor. Assim, as características locais aumentam a acumulação e retenção de água.

O fluxo pipe encontra-se em setores predominante de média vertente. A forma do terreno dominante nessas áreas é divergente-retilíneo, 
apresentando a dispersão do fluxo subsuperficial, dificultando, assim, o aparecimento de canais de drenagem no local, caracterizando, portanto, o predomínio de locais com infiltração de água no solo. Dos cinco pipes observados, o diâmetro entre um e outro variou muito (média de 14,4 $\pm 20,1$ cm). Distingue-se, também, pipes perenes, intermitentes e efêmeros. Dois pipes apresentaram fluxos constantes (pipes perenes), enquanto os outros três apresentaram fluxos que se restringem aos períodos de chuva (pipes intermitentes) ou fluxos apenas durante ou logo após as chuvas (pipes efêmeros).

Nota-se que as conexões hidrológicas existentes dentro do solo nessas áreas, resultaram na transmissão de água entre áreas separadas (topo da vertente) e as redes fluviais (terraço fluvial). Depois de interceptada pelos cortes da estrada, a água do fluxo subsuperficial escoa em velocidade, em razão da pouca resistência (ausência de dissipadores de energia) e pela declividade acentuada (20\%), fazendo com que o fluxo subsuperficial tenha maior volume e competência, sendo então conectado ao canal fluvial (Figura 5b).

O fluxo insaturado prevaleceu nos setores de alta vertente. Essas áreas apresentam uma transição abrupta no setor superior (patamar) com o setor inferior (terraço fluvial). A forma de terreno nesses locais é divergente-convexo que, associado à presença de fragmentos de rocha, resultou na dissipação da água nos locais, principalmente, no sentido longitudinal do talude da estrada rural. O principal efeito observado nessas áreas de interceptação foi que a água tende a se expandir lateralmente no talude quando é aliviada (exfiltrada) por cortes profundo da estrada. Nessa situação, a água é expelida paralela ao talude e na estrada que a intercepta (Figura 5c). 


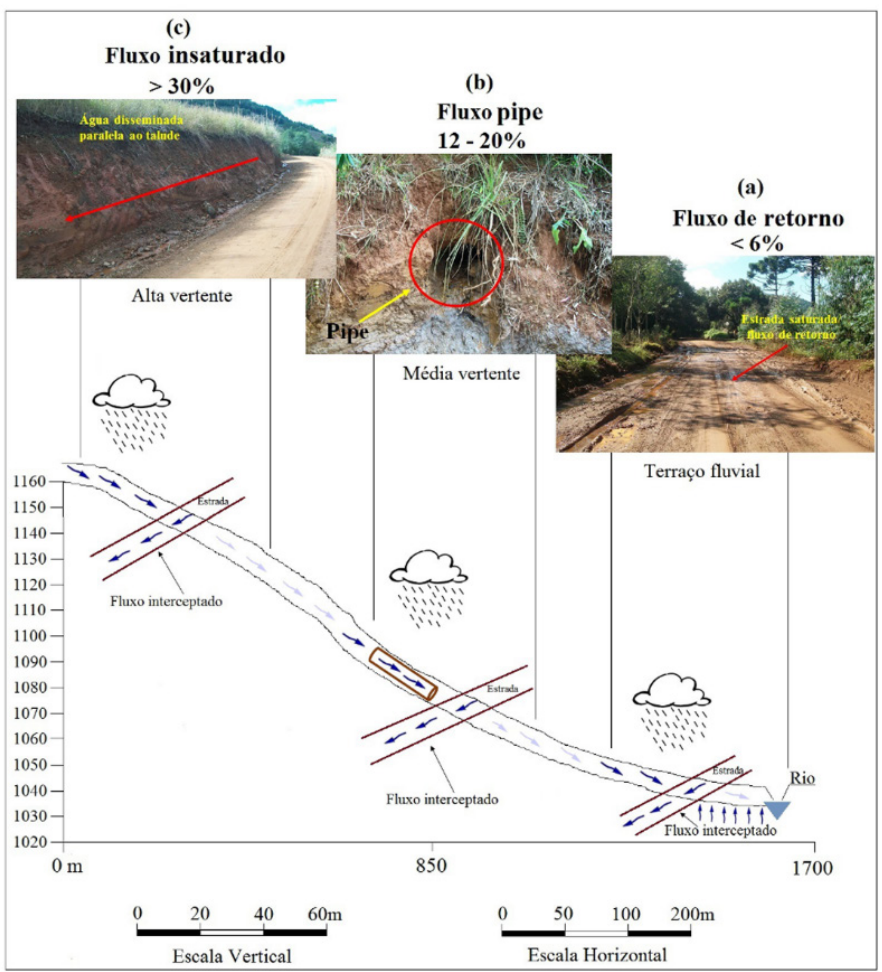

Figura 5 - Modelo conceitual da espacialização dos tipos de interceptação do fluxo subsuperficial, causado por cortes de estradas rurais, de acordo com 0 setor de vertente e a declividade das áreas monitoradas

Fonte: Elaborado pelos autores (2017).

A uniformidade do gradiente topográfico pode beneficiar o sentido da direção do fluxo. Nas áreas de interceptação identificadas foi possível notar um sentido preferencial do movimento da água, mas não um caminho preferencial para o deslocamento em que esse tipo de fluxo se expande vertente abaixo. Como resultado, ocorreram processos erosivos na vertente e na estrada. 
Identificação e caracterização dos tipos de interceptação do fluxo subsuperficial causado por cortes de estradas rurais

O fluxo de retorno é a interceptação de fluxo subsuperficial que ocorre em lençol freático raso ou áreas saturadas, fluxo de retorno próximo à rede de drenagem. Como resultado de uma ação combinada da exfiltração e do fluxo subsuperficial, foi observada a formação de uma camada saturada a pouca profundidade (3 a $15 \mathrm{~cm}$ de espessura), próxima aos cursos fluviais e nos vales com pequena declividade (Figura 6a).

O fluxo pipe é a interceptação de fluxo subsuperficial pontual relacionada à captura de drenagem subterrânea particularmente "pipes" ou dutos. Os pipes são cavidades no solo, com caminhos preferenciais favorecendo a passagem da água com canais de drenagem tubulares no interior do solo. Esses dutos interligados são formados pela ação erosiva dos escoamentos subsuperficiais, que arrastam os sedimentos e também da atividade da fauna e flora. Os mesmos foram encontrados paralelos ao talude da estrada (Figura 6b).

O fluxo insaturado é a interceptação de fluxo subsuperficial difusa (não pontual), por causa da interceptação da água paralela à vertente, formando uma lâmina d'água em média 1 a $3 \mathrm{~cm}$ de altura. Este tipo de fluxo tende a seguir lateralmente os barrancos (cortes mais profundos da estrada), em média $300 \mathrm{~m}$ no sentido longitudinal e responde geralmente às mudanças nos gradientes hidráulicos, resultando em escoamento paralelo ao talude da estrada (Figura 6c). 

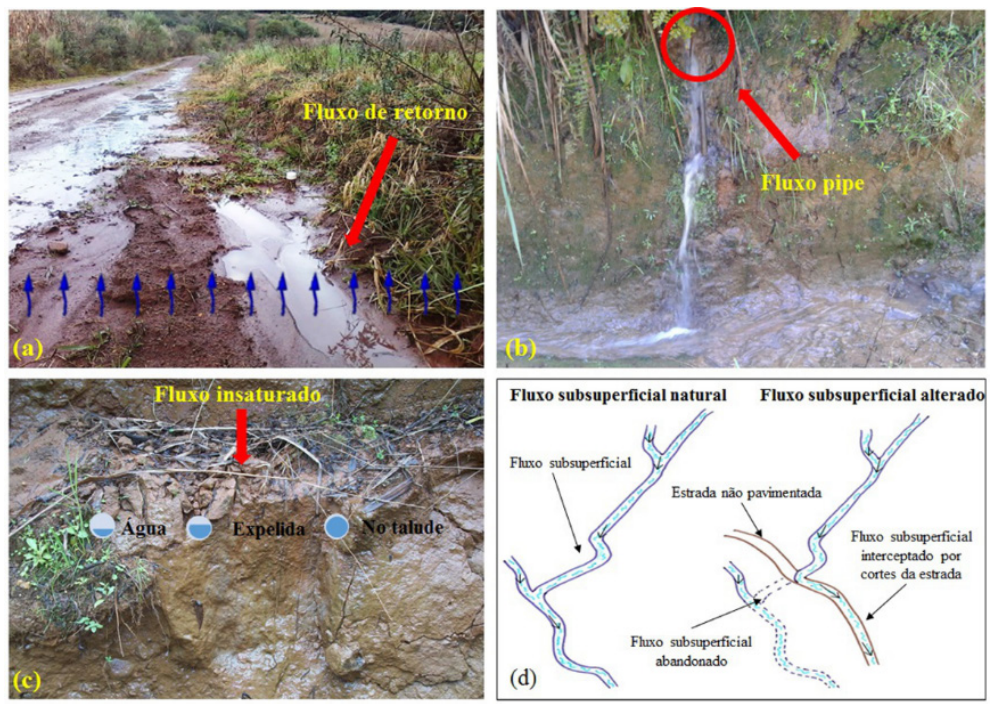

Figura 6 - Características dos tipos de interceptação do fluxo subsuperficial causado por estradas rurais. Em (a) estrada interceptando o fluxo de retorno (saturação prolongada do solo), em (b) estrada interceptando o fluxo pipe (interceptação pontual), em (c) estrada interceptando o fluxo insaturado (água expelida paralela ao talude), e em (d) esquema hipotético da interceptação do fluxo subsuperficial causado por cortes de estradas

Fonte: Adaptado de Furniss, Love e Flanagan (1997).

Observa-se que a inter-relações entre as áreas e os tipos de interceptação resulta primeiramente em interceptação do fluxo subsuperficial pelos cortes da estrada rural (Figura 6d) e, posteriormente, em uma transmissão e redirecionamento desse fluxo concentrado para o canal fluvial (conexão vertente com o rio).

As características de cada tipo de interceptação do fluxo subsuperficial por estradas rurais evidenciaram uma expressiva contribuição com a vazão nos canais fluviais receptores. Como consequência, observou-se que houve confluência entre os três tipos de interceptação do fluxo subsuperficial, resultando assim na conexão com as áreas de alta, média e baixa vertente. 


\section{Discussão}

\section{Distribuição da intercepção do fluxo subsuperficial ao longo da bacia}

$\mathrm{Na}$ área de estudo, as estradas rurais são importantes na interceptação do fluxo subsuperficial, especialmente aquelas mais antigas com taludes mais profundos. A localização das estradas em setores distintos da vertente as tornam ainda mais importantes nas causas de perturbações hidrogeomorfológicas, alterando, assim, a dinâmica natural da bacia. Portanto, as estradas por meio da interceptação do fluxo em subsuperfície afetam o movimento da água e dos sedimentos, contribuindo na dissecação do solo e da vertente, conectando, então, de forma acelerada vertente e rio (Figura 7).

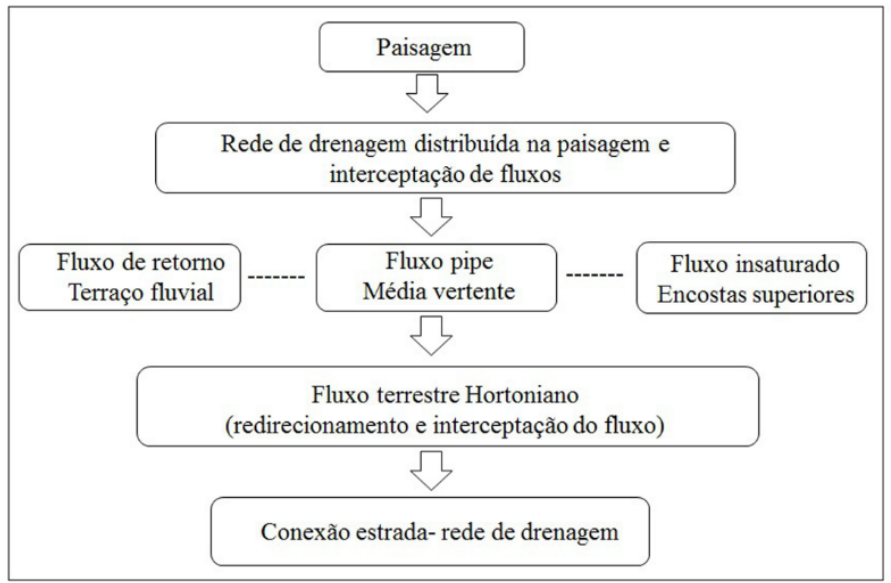

Figura 7 - Modelo conceitual que representa os tipos de interceptação do fluxo subsuperficial causado por cortes de estradas rurais

Fonte: Organizado pelos autores (2017).

Uma clara diferença foi identificada no comportamento dos tipos de interceptação que ocorrem nas áreas em fundo de vale, média e alta vertente. A intercepção do fluxo de retorno ocorre principalmente nas áreas de terraço fluvial próximo à rede de drenagem. Nesse caso, o principal efeito gerado é a saturação prolongada do solo, em razão da dificuldade que a água encontra para infiltrar nas camadas mais profundas. 
Na interceptação do fluxo subsuperficial do fluxo pipe (média vertente) não ocorre dissipação de energia e a água é rapidamente infiltrada. Isso provoca a formação de canais intermitentes na direção longitudinal da estrada, levando à intercepção da subsuperfície de fluxo. Bryan e Jones (1997), Jones (1981), Chappell (2010) advertem que as características do relevo com encostas íngremes, solos permeáveis moderadamente profundos e a formação de dutos são uma condição para o escoamento da água em subsuperfície nesses locais. Esse tipo de interceptação pode apresentar dutos em atividade de poucos centímetros ou dezenas de centímetros de diâmetro com redes que podem se estender por vários metros dentro da encosta.

A intercepção do fluxo insaturado é caracterizada pela predominância nas encostas superiores (Wemple; Swanson; Jones, 2001; Ziegler et al., 2012). Nessa situação, a água é aliviada (expelida) de forma mais lenta nos cortes mais profundo da estrada, onde ocorre a drenagem distribuída paralela à vertente por meio da porosidade existente do solo, que responde, geralmente, às mudanças nos gradientes hidráulicos. Nesse tipo de fluxo subsuperficial, não há uma hierarquia, ou seja, não existe um caminho preferencial único do fluxo, como ocorre no tipo pipe (Terzaghi; Peck, 1966).

Na área de estudo, as estradas são antigas e foram implantadas em diferentes unidades topográficas, não considerando, muitas vezes, a aptidão do terreno (Thomaz; Vestena; Ramos-Scharrón, 2104; Cunha; Thomaz, 2015). E por causa da natureza linear das estradas e sua tendência em cruzar diferentes gradientes topográficos, principalmente a rede de drenagem, afetam, sobretudo, o movimento da água e dos sedimentos (Luce; Wemple, 2001; Ziegler, 2012).

Como consequência ocorrem problemas como rampas acentuadas de difícil trafegabilidade, encostas instáveis e de difícil manutenção, expondo, muitas vezes, perfis do solo susceptíveis à erosão. Nesse caso, as estradas são encaixadas na encosta, com taludes expostos, apresentando também pista sem controle de drenagem superficial e subsuperficial, ausência de vegetação em áreas de entorno e cruzamentos de estradas e rios (Wemple; Vestena; Ramos-Scharrón, 2001; Luce, 2002; Forman; Sperling; Bissonette, , 2003; Cunha; Thomaz, 2015; Thomaz; Peretto, 2016). 
Características do fluxo subsuperficial interceptado por cortes de estradas rurais

Na área estudada, nota-se que as estradas rurais estão conectadas hidrologicamente aos canais fluviais por meio dos cruzamentos, que servem como pontos de entrada para o fluxo subsuperficial concentrado. Esses cruzamentos de estradas e rios ocorrem de forma distribuída na bacia, entretanto, nos terraços fluviais (estradas principais) se intensificam mais.

Nas áreas saturadas, acontece forte conectividade entre os fluxos interceptados no declive superior (fluxo insaturado), resultando na soma do fluxo de subsuperfície interceptado em média vertente (fluxo pipe) e o fluxo interceptado em fundo de vale (fluxo de retorno). Luce e Wemple (2001) e Bracken et al. (2013) enfatizaram que esse excesso de fluxo subsuperficial interceptado é redirecionado para os canais fluviais, possivelmente contribuindo para os picos de vazão.

Em geral, mais de 95\% da chuva que cai sobre uma bacia hidrográfica precisa passar por um divisor topográfico antes de ser transferida para um canal fluvial (Knighton, 1998). Além disso, a água pode seguir vários caminhos do divisor topográfico até chegar ao rio, por exemplo, o fluxo terrestre Hortoniano, o fluxo de retorno, o fluxo insaturado e saturado e o fluxo subsuperficial pipe.

A resposta da captação aos processos de fluxo da encosta é dependente do tipo de processo envolvido na transferência de água. Infiltração ou excesso de fluxo terrestre pode gerar um hidrograma de tempestade em poucas horas ou minutos após um evento de chuva. No entanto, o fluxo de tempestade subsuperficial exibirá um tempo de latência de muitas horas ou dias para gerar um hidrograma de tempestade. Em suma, o escoamento superficial é mais rápido do que o fluxo subsuperficial (Anderson; Burt, 1990).

Assim, as estradas rurais transformam todo o fluxo subsuperficial interceptado em fluxo Hortoniano, isto é, o escoamento superficial é produzido, porque a intensidade de chuva excede a capacidade de infiltração de água no solo. Além disso, na área de estudo durante tempestades, o leito da estrada gera importante fluxo superficial variando de $17 \%$ a 58\% (Thomaz; Ramos-Scharrón, 2015). A velocidade do fluxo Hortoniano varia entre 30 m/h-1 e 500 m/h-1 (Knighton, 1998). Portanto, a rede viária é crucial na resposta da captação do hidrograma, porque 
transforma a maior parte dos processos de fluxo lentos de água para processos de fluxo mais rápido.

Em certa escala, a conectividade hidrogeomórfica nas estradas que cruzam os rios podem afetar a dinâmica do fluxo natural, que pode ser completamente controlado pelos processos hidrogeomorfológicos da rede viária (Thomaz; Peretto, 2016). Portanto, os tipos de interceptação do fluxo subsuperficial têm diferentes mecanismos de funcionamento. Esses mecanismos diferenciados do funcionamento hidrológico de áreas interceptadas podem alterar a dinâmica natural da bacia hidrográfica, produzindo, então, resposta hidrológica menor que por meio da infiltração e transferência de fluxo interno no solo conectando de forma acelerada vertente e rio.

\section{Conclusão}

Geralmente, as estradas não pavimentadas transformam o fluxo subsuperfical em fluxo terrestre Hortoniano. As áreas de interceptação de fluxo subsuperficial estão distribuídas em diferentes setores de declividade. O fluxo de retorno é observado, principalmente, em fundo de vale e áreas úmidas onde a intercepção do fluxo ocorre em lençol freático raso ou áreas saturadas próximas à rede de drenagem. O fluxo pipe prevalece, em média vertente, possuindo um caminho preferencial único que favorece a passagem da água até ser interceptado por cortes da estrada. O fluxo insaturado distribui-se com mais frequência em alta vertente encaixado no talude da estrada, sendo exfiltrada lateralmente.

Os três tipos de fluxos identificados apresentam propriedades específicas. O fluxo de retorno é resultado da exfiltração da água do solo e a soma do fluxo subsuperficial interceptado. O fluxo pipe é a interceptação que ocorre, por meio de dutos interligados no solo, com caminho preferencial único. Finalmente, o fluxo insaturado ocorre quando a água é disseminada paralela ao talude da estrada.

As estradas rurais desempenham um papel fundamental na ligação de áreas separadas na área de estudo, alterando, assim, a resposta hidrológica natural da bacia hidrográfica. Estas estradas interceptam o fluxo subsuperficial e o redirecionam para os canais fluviais, resultando em um tempo de resposta hidrológica mais rápido em comparação com o fluxo de água subsuperficial transferido por meio do solo. 


\section{Referências}

ANDERSON, M.; BURT, T. Process Studies in Hillslope Hydrology. John Wiley and Sons: Chichester, 1990. p. 539.

ATLAS geológico do estado do Paraná. Curitiba: MINEROPAR, 2001. Disponível em: <http://www.mineropar.pr.gov.br/arquivos/File/MapasPDF/atlasgeo.pdf>. Acesso em: 25 set. 2017.

BAARTMAN, J. E. et al. Linking landscape morphological complexity and sediment connectivity. Earth Surf. Process. Landf., v. 38, n. 12, p. 1457-1471, 2013. doi:10.1002/esp.3434

BRACKEN, L. J.; CROKE, J. The concept of hydrological connectivity and its contribution to understanding runoff-dominated geomorphic systems. Hydrol. Process.,v. 21, n. 13, p.1749-1763, 2007. Disponível em: < http://onlinelibrary. wiley.com/doi/10.1002/hyp.6313/pdf>. Acesso em: 23 jul. 2017.

BRACKEN, L. J. et al. Concepts of hydrological connectivity: Research approaches, pathways and future agendas. Earth-Science Reviews., v. 119, n. 18, p. 17-34, 2013. Disponível em: <https://doi.org/10.1016/j.earscirev.2013.02.001>. Acesso em: 23 jul. 2017.

BRYAN, R. B.; JONES, J. A. A. The significance of soil piping processes: inventory and prospect. Soil Erosion Laboratory, University of Toromo, Scarborough, Ont. M1C IA4, Canadá. Geomorphology., v. 20, n. 4, p. 209-218, 1997. Disponível em: <https://doi.org/10.1016/S0169-555X(97)00024-X>. Acesso em: 23 jul. 2017.

BRYCESON, D. F.; BRADBURY, A.; BRADBURY, T. Roads to Poverty Reduction? Exploring Rural Roads' Impact on Mobility in Africa and Asia. Development Policy Review v. 26, n.4, p. 459-482, 2008. doi:10.1111/j.1467-7679.2008.00418.x.

CAVIGLIONE, J. H. et al. Cartas climáticas do Paraná. Londrina: IAPAR-Instituto Agronômico do Paraná, 2000.

CHAPPELL, N. A. Soil pipe distribution and hydrological functioning within the humid tropics: a synthesis. Hydrol. Process., v. 24, n.12, p. 1567-1581, 2010. doi:10.1002/hyp.7634

CHENG, B. et al. Constructing China's roads as works of art: a case study of 'esthetic greenway' construction in the Shennongjia region of China. Land Degradation and Development., v. 26, n. 4, p. 324-330, 2015. doi: 10.1002/ldr.2210.

CUNHA, M. C.; THOMAZ, E. L. É possível reduzir a turbidez da água em bacia rural por Meio de implantação de caixas de infiltração? Revista Brasileira de Geomorfologia, São Paulo, v. 16, n. 4, p. 657-667, 2015. Disponível em: <http:// dx.doi.org/10.20502/rbg.v16i4.725>. Acesso em: 23 jul. 2017.

ESRI. Environmental Systems Research Institute. ArcGIS 10.2. [2013]. Disponível em:< https://www.esri.com/en-us/home>. Acesso em: 25 set. 2017.

FAIZ, A. et al. Sustainable rural roads for livelihoods and livability. Procedia Social and Behavioral Sciences., v. 53, n.1, p. 1-8, 2012. Disponível em: <https:// doi.org/10.1016/j.sbspro.2012.09.854>. Acesso em: 23 jul. 2017. 
FORMAN, R. T.; SPERLING.; D. BISSONETTE, J. A. et al. Road Ecology. Washington: Island Press, 2003.

FURNISS, M. J.; LOVE, M.; FLANAGAN, S. A. Diversion Potential at RoadStream Crossings. United States Department of Agriculture Forest Service Technology e Development Program, 1997.

IUSS WORKING GROUP WRB. World reference base for soil resources a framework for international classification, correlation and communication. World Soil Resources Reports 103. Food and Agriculture Organization of the United Nations, Rome, 2006.

JAIROSI, I. Y. Digital Biodiversity Data Standards e Guidelines. Southern African Biodiversity Support Programme, 2001.

JONES, A. A. The Nature of Soil Piping, a Review of Research. Norwich: Geobooks, 1981. p. 301.

KNIGHTON, D. Fluvial Forms and Processes: A New Perspective. New York: Arnold, 1998.

KÖPPEN, W. Climatologia. México: Fundo de Cultura Econômica, 1948.

LUCE, C. H. Hydrological processes and pathways affected by forest roads: what do we still need to learn? Hydrol. Process., v.16, n.14, p. 2901-2904, 2002. doi:10.1002/hyp.5061.

LUCE, C. H.; WEPLE, B. C. Introduction to special issue on hydrologic and geomorphic of forest roads. Earth Surf. Process. Landf., v. 2, n.8, p. 111-113, 2001. doi:10.1002/1096-9837(200102)26:2<111::AID-ESP165>3.0.CO;2-2.

SIDLE, R. C.; ZIEGLER, A. D. The dilemma of mountain roads. Nature Geoscience., v. 5, n.7, p. 437-438, 2012.

TERZAGHI, K.; PECK, R. B. Soil Mechanics in Engineering Practice. New York: Wiley, 1966. p. 566.

THOMAZ, E. L.; PERETTO, G. T. Hydrogeomorphic connectivity on roads crossing in rural headwaters and its effect on stream dynamics. Science of the Total Environment., v. 550, n. 1, p. 547-555, 2016. Disponível em: <https:/doi. org/10.1016/j.scitotenv.2016.01.100>. Acesso em: 23 jul. 2017.

THOMAZ, E. L.; RAMOS-SCHARRÓN, C. E. Rill length and plot-scale effects on the hydrogeomorphologic response of gravelly roadbeds. Earth Surf. Process. Landf.,v.40, n.15, p. 2041-2048, 2015. doi: 10.1002/esp.3778.

THOMAZ, E. L.; VESTENA, L. R.; RAMOS-SCHARRÓN, C. E. The effects of unpaved roads on suspended sediment concentration at varying spatial scales a case study from Southern Brazil. WEJ (Hertford).,v. 28 n.4, p. 547-555, 2014. doi:10.1111/wej.12070.

WEMPLE, B. C.; SWANSON, F. J.; JONES, A. A. Forest roads and geomorphic process interactions, Cascade Range, Oregon. Earth Surf. Process. Landf., v. 26, n.2, p. 191-204, 2001. Disponível em: <http://dx.doi. org/10.1002/10969837(200102)26:2b191::aid-esp175N3.0.co;2-u>.Acesso em: 23 jul. 2017. 
ZIEGLER, A. D. et al. Towards better design and management of tsunami evacuation routes: a case study of a Jak Beach Road. Geol. Soc. Special. Publ. London, v. 361, n. 4, p. 107-114, 2012. Disponível em:< http://dx.doi.org/10.1144/ SP361.9>. Acesso em: 23 jul. 2017.

Márcia Cristina da Cunha - possui graduação em Geografia pela Faculdade Estadual de Ciências e Letras de Campo Mourão, Mestrado em Geografia pela Universidade Estadual do Centro-Oeste, e Doutorado em Geografia Física pela Universidade Federal do Paraná (UFPR). Atua como professora colaboradora desde 2007 no Departamento de Geografia, Universidade Estadual do Centro-Oeste.

Edivaldo Lopes Thomaz - graduado em Geografia pela Universidade Estadual de Londrina. Mestre e Doutor em Ciências, Geografia Física, pela Universidade de São Paulo com estágio na University of Toronto, Canadá. Pós-doutor em Geomorfologia pela The Universtiy of Texas at Austin, Estados Unidos. Atualmente é Professor Associado no Departamento de Geografia da Universidade Estadual do Centro-Oeste.

\section{Contribuições dos autores}

Os autores ofereceram substanciais contribuições científicas e intelectuais ao estudo. $\mathrm{O}$ autor Edivaldo Lopes Thomaz ficou especialmente responsável pelo desenvolvimento teórico-conceitual e apoio no processamento dos dados; a autora Márcia Cristina da Cunha ficou responsável pela aquisição de dados e seu processamento, interpretação e análise dos mesmos. 